\title{
Role of Smoking in the Pathogenesis of Multiple Sclerosis: A Review Article
}

Katukuri Nishanth $^{1}$, Ezza Tariq ${ }^{1,2}$, Farirai P. Nzvere ${ }^{1}$, Mohammed Miqdad ${ }^{1}$, Ivan Cancarevic ${ }^{1}$

1. Internal Medicine, California Institute of Behavioral Neurosciences \& Psychology, Fairfield, USA 2. Internal Medicine, Nishtar Medical College, Multan, PAK

Corresponding author: Katukuri Nishanth, katukurinishanth553@gmail.com

\begin{abstract}
Multiple sclerosis (MS) is a complex and unpredictable neurological condition. It is the most commonly seen autoimmune disorder. The incidence of disease and its prevalence are growing worldwide. Early identification of the disease and accurate diagnosis is important to prevent further complications and disability. The etiology remains unclear, and it is believed that complex gene-environment interactions play an essential role. Genetic predisposition only describes a portion of the disease risk, whereas lifestyle and environmental factors are significant contributors. Smoking was identified as an important risk factor for MS. The main objectives of this review were to examine the underlying mechanisms of immune dysregulation in the development of MS, explore the association between smoking and MS, and identify other genetic and environmental factors that alter the risk of developing the disease. We searched PubMed for articles relevant to the study topic published between 2000 and 2020 using the search terms "multiple sclerosis," "cigarette smoking," "risk factors," and, "epigenetics." Studies reveal a marked association between smoking and the risk of MS. Unlike genetic risk factors, many lifestyles and environmental factors can be adjusted, with potential for prevention, particularly for people at the highest risk, such as families of individuals with MS.
\end{abstract}

Categories: Family/General Practice, Internal Medicine, Neurology

Keywords: cigarette smoking, multiple sclerosis, epigenetics, epidemiology, risk factors

\section{Introduction And Background}

Multiple sclerosis (MS) is the most common autoimmune inflammatory demyelinating disorder of the brain and spinal cord [1]. It is a widespread debilitating and disabling neurological disease commonly seen in young adults worldwide [2]. Most often, people with MS are diagnosed between 20 and 40 years of age. MS patients commonly experience urinary symptoms, pain, sexual problems, tiredness, visual disturbances, muscle stiffness, spasticity, changes in cognitive function, and depression. The course of the disease is very unpredictable in such a way that a small number of patients with MS have a mild course of infection with little to no disability; on the other hand, some people develop a steadily worsening disease that leads to increased disability over time. Environmental factors like smoking, low vitamin D, Epstein-Barr virus (EBV) infection, childhood obesity, and lack of physical activity are some of the risk factors for MS [1]. Four basic

Received 06/15/2020 Review began 06/25/2020 Review ended 07/24/2020 Published 08/05/2020

\section{() Copyright 2020}

Nishanth et al. This is an open access article distributed under the terms of the Creative Commons Attribution License CC-BY 4.0., which permits unrestricted use, distribution, and reproduction in any medium, provided the original author and source are credited. disease courses have been identified in MS: clinically isolated syndrome (CIS), primary progressive MS (PPMS), relapsing-remitting MS (RRMS), and secondary progressive MS (SPMS) [3].

CIS refers to the first acute episode suggestive of inflammation and demyelination in the central nervous system (CNS) [3]. CIS may be the first presentation of the illness. The risk of developing MS following CIS is very high. When CIS is associated with lesions on brain MRI similar to the ones seen in MS, there is a higher chance for a second neurological event and diagnosis of clinically definite MS (CDMS). Individuals who experience CIS alone with no evidence of MS-like lesions on brain MRI are at relatively low risk of developing MS. RRMS affects about $85 \%$ of patients and is defined by relapses lasting from days to weeks, followed by complete or partial remission over months or years [1]. PPMS affects approximately $10 \%$ of the patients. PPMS is marked by a progressive accumulation of disabilities following the disease's initial relapse; symptoms tend to deteriorate and become more resistant to drug therapies [1-3]. SPMS follows an initial relapsing-remitting path [1]. Nearly 75\% of patients with RRMS will transition to SPMS within 15 years of initial diagnosis [4]. Besides progressive worsening of neurological functions, treatment with diseasemodifying agents may delay the progression of RRMS to SPMS [1,4].

Currently, there is no cure, and researchers are still trying to find the cause of the disease [2]. Both genetic and environmental factors play an important role in developing MS [5]. New medicines and therapies developed in the last few years show promising results in slowing down the progression of the disease, disability, and complications [2]. Researchers see promising results with drugs and therapies that are designed to protect myelin cells against damage or that could help them recover after injury. On 25 March 2020, US Food and Drug Administration approved drug Zeposia (ozanimod) oral capsules to treat adults with relapsing forms of MS [6]. NINDS (National Institute of Neurological Disorders and Stroke) sponsored a clinical trial to know whether coupling two therapies, glatiramer acetate, and beta-interferon are beneficial 
for preventing relapses [2].

The number of MS cases has significantly increased since 1990, and recent studies estimate that more than 2.5 million people have this condition globally [2]. Nearly 250,000-300,000 people diagnosed with MS in the United States. Environmental factors, like smoking, are risk factors for the development, severity, progression, disability, and early death in MS [5,7-9]. In this review, we are going to discuss MS potential etiological factors, epidemiology, and pathology.

\section{Review}

\section{Damaging effects of cigarette smoke on the immune system in MS}

The effects of cigarette smoke on the immune system are not well known [10]. Tobacco contains high amounts of free radicals. It has been reported that free radical-induced oxidative stress causes mutations in genetic material and is involved in several neurodegenerative disorders, such as Parkinson's disease and MS. Tobacco increases the risk of autoimmune diseases, such as systemic lupus erythematosus and rheumatoid arthritis, and is associated with other autoimmune disorders like MS [11,12]. Cigarette smoke acts on the cellular level of the immune system, resulting in the development of proinflammatory cytokines [11-13]. Smokers have high levels of proinflammatory cytokines (e.g., IL-6), higher levels of C-reactive protein, fibrinogen, and other inflammatory markers [11]. Sustained, elevated levels of proinflammatory cytokines could contribute to persistent autoimmunity. In general, people with MS have higher levels of NO, due to the presence of an inducible form of nitric oxide synthase (iNOS) in cells, such as macrophages and astrocytes [14]. The elevated levels of NO can cause mitochondrial damage, oligodendrocyte necrosis, and axonal degeneration, ultimately leading to impairment in axonal conduction [14,15]. Exposure to harmful gases like carbon monoxide (CO) affects tissue oxygenation and can lead to demyelination [7]. Carbon monoxide (CO) neurotoxicity occurs through a number of mechanisms including lipid peroxidation, inflammatory, myelin degeneration, immune-mediated reactions, and finally neuronal apoptosis and necrosis. Smoking decreases the numbers of peripheral B cells and natural killer (NK) cells, decreases the production of cytokines by antigen-presenting cells, and reduces the blood levels of serum immunoglobulins [11]. Nitric oxide (NO) and carbon monoxide (CO), in cigarette smoke, enhance the inflammatory response and attenuate some of the immune defenses resulting in increased susceptibility to infections [7,13]. These changes in the immune system explain a few possible mechanisms for increased susceptibility to viral respiratory tract infections in MS patients $[7,11,13]$. Respiratory infections are an essential trigger for relapse in MS [11]. Sibley et al. found the risk of relapse was increased almost threefold in two weeks prior and three weeks post-respiratory viral infection [16]. Tobacco smoking lessens the activation of $\mathrm{CD} 4^{+} \mathrm{T}$ cells, modifies antigen-mediated T-cell signaling, and causes inactivation of $\mathrm{CD}^{+} \mathrm{T}$ cells in response to infection [17].

Additionally, smoking increases the expression of Fas (CD95), a cell surface molecule on CD $4^{+}{ }^{-}$cells and B cells, and makes these cells more sensitive to apoptosis [18]. Increased accumulation of apoptotic material may be significant if clearance mechanisms are weakened in individuals with autoimmunity. Cigarette smoke induces irritation in the lung and may trigger the development of autoaggressive T cells in the lungs, which are cross-reactive with CNS antigens and initiate a CNS-directed autoaggressive immunity resulting in MS [19]. In the tobacco, a polyphenol-rich glycoprotein stimulates the proliferation of T cells and differentiation of B cells, creating a proinflammatory environment [7]. Proinflammatory cells like G-proteincoupled receptor 15 (GPR15) T cells are increased in smokers and associated with MS [20]. Acrolein and hydrogen cyanide in cigarette smoke can induce immunosuppression and neurodegeneration [7,15].

To sum up, smoking is a major environmental risk factor for the incidence of MS, and it plays a vital role in immunological mechanisms. Data from multiple studies show that tissue injury in MS is due to an abnormal immune response to one or more myelin antigens that develops in genetically susceptible individuals after exposure to the risk factors like cigarette smoke. Therefore, stopping smoking could alleviate atypical immune responses and tissue damage.

\section{Epigenetics of MS}

Epigenetics play a crucial role in the pathogenesis of MS [21]. Current research implies that the interplay between genetic and environmental factors is necessary for disease manifestation [22]. The smoking status may strongly influence the risk of developing MS associated with human leukocyte antigen (HLA) genotypes [23]. HLA-DRB1*15:01 and HLA-A*02 are considerable risk and protective variants for MS, respectively $[15,19]$. Smokers carrying HLA-DRB1*15 and lacking HLA-A*02 had a 14-fold increased risk compared with non-smokers $[15,19,23]$. The inheritance pattern of MS is still unknown, although the condition appears to pass down through generations in families [24]. Incidence and prevalence of MS are higher among the siblings or children of a person with the disease than for the general population. There is a higher clinical concordance rate (25\%-30\%) among monozygotic or identical twins than dizygotic or fraternal twins (3\%7\%) [25]. HLA-DRB1*15:01, genetic risk score (GRS), and smoking were associated with earlier onset of disease [26]. A significant interaction between NAT1 variant rs7388368, HLA-DRB1*15:01, and HLA- A*02 was observed among smokers [23,27]. In contrast, such an association was absent among non-smokers [23]. Genome-wide association studies (GWAS), a new study design introduced in the mid-2000s, revolutionized the genetics of MS [22]. To date, the most extensive genetic study has identified 233 genome-wide loci 
associated with MS susceptibility [25]. Out of 233 loci, 200 of these loci explain 20\% of the genetics [25]. Increased or decreased acetylation, methylation, and citrullination of genes controlling the expression of inflammation and myelination factors appear to be mainly involved [21]. HLA-DRB1*15:01 allele is the significant risk factor for MS and regulated by epigenetic mechanisms, such as DNA methylation and histone deacetylation. Some pieces of evidence support that blood DNA methylation is affected by cigarette smoke [28]. Smoking causes DNA methylation in MS patients in an exposure-dependent manner [23,28]. Hypomethylation is more pronounced in current smokers or those who ceased smoking less than five years ago [28]. In comparison, methylation levels in MS patients who stopped smoking more than five years ago are comparable to those in non-smokers.

Genetic predispositions, along with environmental factors, play an essential role in the pathogenesis of the disease. The wide heterogeneity in etiology, together with the varying responses to treatment, signifies further hurdles to understand and treat the underlying condition. Over the last decade, we have seen real progress in defining the genetic basis of MS. Identifying and characterizing MS susceptibility genes and their correlation with disease phenotypes is likely to identify the underlying etiology of the disease, improve risk assessment, and influence therapeutics.

\section{Anatomical and cognitive deficits in MS patients}

Smoking affects the cognitive functions of MS patients [29,30]. The prevalence of cognitive deficits in patients with MS ranges from 40\% to 70\% [29]. Memory is most often affected, and the number of patients that experience memory and learning problems is between $40 \%$ and $60 \%$ [31]. Cognitive deficits may occur depending on the size and location of the focal area of demyelination in the CNS [30]. Studies have reported that male MS patients have more significant cognitive impairments than females, but in recent research, it was found that sex is not associated with cognitive impairment [32,33]. A few studies were conducted to determine if nicotine or the tar in tobacco smoke was responsible for the adverse effects [30]. These studies concluded that nicotine had positive effects on cognition. Exposure to high concentrations of tar in cigarette smoke may be causing cognitive problems in MS patients. Heavy smokers had increased cognitive impairment when compared to non-smokers. Brain MRI obtained from smokers with MS showed a higher number of plaques, reduced gray matter fraction, lower brain parenchymal fraction, and increased cerebrospinal fluid fraction compared to those who have never smoked or stopped smoking [34,35]. In contrast, no effect was observed on white matter fraction [34]. A decrease in brain volume, especially gray matter, is a strong predictor of long-term physical disability and cognitive issues.

Cigarette smoking has both positive and negative cognitive effects, and due to the increasing incidence of cognitive impairment in MS patients, it is important to explain the cognitive effects of smoking in these patients. Brain volume measures will likely be used as predictors of treatment response and disease progression. They will also have a significant impact on treatment decisions, mainly to the development of new MS drugs that could prevent brain atrophy. Understanding decreases in brain matter can become necessary for MS management. Therefore, patients should be advised to avoid smoking to reduce exposure to substances such as tar in cigarette smoke.

\section{Smoking and disease progression in MS}

Smoking adversely influences the course of the disease [7,36]. According to the studies mentioned in Table 1 , smokers have an elevated risk of developing MS compared to non-smokers [37-40]. Research suggests that both the duration (years) and intensity of tobacco smoking also affect the risk of developing the disease $[40,41]$. Current cigarette smokers had more severe disease at baseline than never-smokers in terms of Expanded Disability Status Scale score (EDSS) [37]. Exposure to pollutants present in tobacco-related products contributes to the development of proangiogenic and pathogenic CCR6 ${ }^{+}$Th17 cells [12]. These cells worsen chronic inflammatory disorders, such as MS and rheumatoid arthritis [12]. Di Pauli et al. study reported that nicotine users diagnosed with the CIS develop a clinically definite course of the disease (CDMS) in a shorter period than non-smokers [42]. A large prospective cohort study conducted by Healy et al. suggests that patients who quit smoking may not only decrease the risk of developing smoking-related complications but also delay the progression of MS [37]. Patients with this condition are sensitive to the central effects of smoking, resulting in an acute worsening of symptoms like blurred vision, motor weakness, and paresthesias [36]. Cigarette users diagnosed with this autoimmune disorder experience more severe symptoms, an increased number of relapses, and more significant disability compared to non-smokers [43]. Data from multiple studies suggest that cigarette smoke has an influence on disease progression and accelerates the conversion from a relapsing-remitting to a progressive course (SPMS) of MS [37,43,44]. Ramanujam et al. found that the risk for transition to a secondary progressive form of the disease was increased by $4.7 \%$ per year for each additional year of smoking after the diagnosis of RRMS [45]. Pittas et al. stated patients who had ever smoked were twice likely to develop the progressive form of the condition [44]. Maghzi et al. found that an ex-smoking habit could increase the risk of MS by 8.83-fold [46]. Sundström and Nyström reported that people who started smoking early ( $<15$ years of age) were more likely to develop progressive forms of the disease [47]. In a three-year prospective cohort study conducted in southern Tasmania, it was determined that using cigars was positively associated with clinical disability progression [44]. 


\section{Cureus}

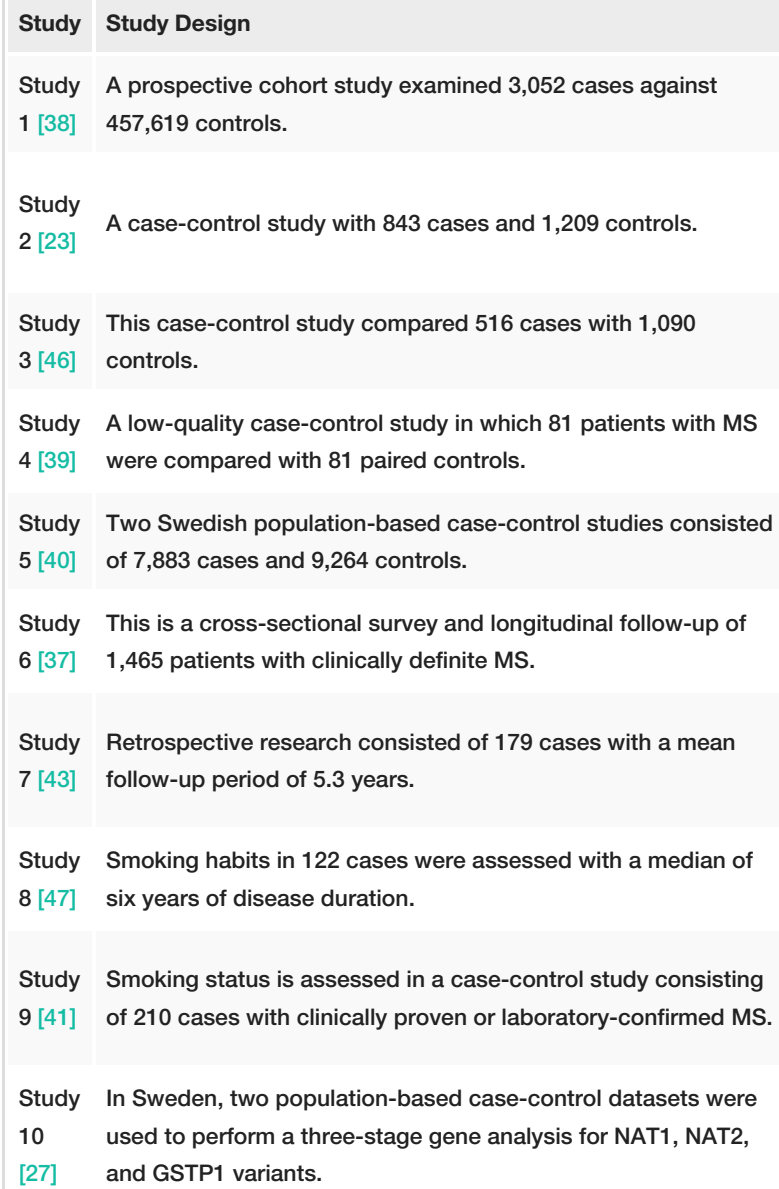

Study A low-quality case-control study in which 81 patients with MS

4 [39] were compared with 81 paired controls.

Study Two Swedish population-based case-control studies consisted

5 [40] of 7,883 cases and 9,264 controls.

Study This is a cross-sectional survey and longitudinal follow-up of

6 [37] 1,465 patients with clinically definite MS.

Study Retrospective research consisted of 179 cases with a mean

7 [43] follow-up period of 5.3 years.

Study Smoking habits in 122 cases were assessed with a median of

8 [47] six years of disease duration.

Study Smoking status is assessed in a case-control study consisting

9 [41] of 210 cases with clinically proven or laboratory-confirmed MS.

Study In Sweden, two population-based case-control datasets were 10 used to perform a three-stage gene analysis for NAT1, NAT2,

[27] and GSTP1 variants.

\section{Main Outcome}

Reported a relative risk (RR) of 1.48 and concluded that smoking might increase the susceptibility to multiple sclerosis (MS)

Among those with genetic risk factors, smoking increased the risk by a factor of 2.8 in comparison with a factor of 1.4 among those without the genetic risk factors.

Past smokers and current smokers had a significant risk of developing MS.

This study stated that ever smoking could increase the risk of MS by 7.6-fold.

This research saw a clear dose-response association between cumulative dose of smoking and MS risk.

MS in smokers progressed from relapsing-remitting (RRMS) to secondary progressive disease (SPMS) faster than in never-smokers.

These results suggest that smoking may be a risk factor for transforming a relapsing-remitting (RRMS) course into a secondary progressive course (SPMS).

Compared with never-smokers, ever-smokers were more likely to have a progressive illness.

A dose-response relationship between the risk of MS and both duration (years) of smoking and the number of cigarettes smoked daily was observed.

They found out NAT1 as a genetic effect modifier of tobacco smoke exposure in MS susceptibility.

TABLE 1: Key studies on the association between smoking and MS.

Studies described in Table 1 provide clinicians with valuable data by showing that individuals affected by MS who are actively smoking are more likely to experience a more severe clinical course with significant deficits in all domains. Stopping tobacco use could delay the onset of symptoms, disability development (use of a walker, or assistive device), and reduce the number of relapses and complications.

\section{Conclusions}

MS is a complex neurological disease in which the interplay between genetic and environmental factors triggers a cascade of events, including activation of the immune system, CNS demyelination, and neuronal degeneration with variable degrees of repair. In this article, we have analyzed the association between smoking and MS in different population groups. Smoking should be considered as a modifiable risk factor for MS, and it may account for some of the excess mortality among patients with MS. The development of therapeutics incorporating neuroprotection and remyelination to treat and eventually prevent the disabling and progressive forms of the condition is a challenge. Early diagnosis and treatment could help prevent the complications and disability associated with MS. Health promotions aimed at smoking cessation must be encouraged. More research needs to be done to improve our understanding of the possible links between tobacco and MS, to investigate the harmful effects of tobacco on mentation, to determine the impact of exposure to second-hand smoke, and to assess whether smoking affects the patient's response to immunomodulatory therapy.

\section{Additional Information \\ Disclosures}

Conflicts of interest: In compliance with the ICMJE uniform disclosure form, all authors declare the following: Payment/services info: All authors have declared that no financial support was received from any organization for the submitted work. Financial relationships: All authors have declared that they have no financial relationships at present or within the previous three years with any organizations that might have an interest in the submitted work. Other relationships: All authors have declared that there are no other relationships or activities that could appear to have influenced the submitted work. 


\section{References}

1. Goldenberg MM: Multiple sclerosis review. P T. 2012, 37:175-184.

2. Multiple sclerosis: hope through research. (2012). Accessed: May 3, 2020: https://www.ninds.nih.gov/Disorders/Patient-Caregiver-Education/Hope-Through-Research/MultipleSclerosis-Hope-Through....

3. Types of MS. (2019). Accessed: May 12, 2020: http://www.nationalmssociety.org/What-is-MS/Types-of-MS.

4. The pathologic foundations of multiple sclerosis: current considerations . (2019). Accessed: May 15, 2020: https:/www.ajmc.com/journals/supplement/2019/role-brain-preservation-management-ms/thepathologic-foundations-of-mul...

5. Wingerchuk DM: Smoking: effects on multiple sclerosis susceptibility and disease progression . Ther Adv Neurol Disord. 2012, 5:13-22. 10.1177/1756285611425694

6. Drug trials snapshots: ZEPOSIA. (2020). Accessed: May 9, 2020: https://www.fda.gov/drugs/developmentapproval-process-drugs/drug-trials-snapshots-zeposia.

7. Shirani A, Tremlett H: The effect of smoking on the symptoms and progression of multiple sclerosis: a review. J Inflamm Res. 2010, 3:115-126. 10.2147/JIR.S12059

8. Hernán MA, Olek MJ, Ascherio A: Cigarette smoking and incidence of multiple sclerosis . Am J Epidemiol. 2001, 154:69-74. 10.1093/aje/154.1.69

9. Jasielski P, Piędel F, Rocka A, Petit V, Rejdak K: Smoking as a risk factor of onset and relapse of multiple sclerosis: a review. Neurol Neurochir Pol. 2020, 54:243-251. 10.5603/PJNNS.a2020.0032

10. Pryor WA, Stone K: Oxidants in cigarette smoke. Radicals, hydrogen peroxide, peroxynitrite, and peroxynitrite. Ann N Y Acad Sci. 1993, 686:12-27. 10.1111/j.1749-6632.1993.tb39148.x

11. O'Gorman C, Lucas R, Taylor B: Environmental risk factors for multiple sclerosis: a review with a focus on molecular mechanisms. Int J Mol Sci. 2012, 13:11718-11752. 10.3390/ijms130911718

12. Baskara I, Kerbrat S, Dagouassat M, et al.: Cigarette smoking induces human CCR6+Th17 lymphocytes senescence and VEGF-A secretion. Sci Rep. 2020, 10:6488. 10.1038/s41598-020-63613-4

13. Alrouji M, Manouchehrinia A, Gran B, Constantinescu CS: Effects of cigarette smoke on immunity, neuroinflammation, and multiple sclerosis. J Neuroimmunol. 2019, 329:24-34.

10.1016/j.jneuroim.2018.10.004

14. Smith KJ, Lassmann H: The role of nitric oxide in multiple sclerosis . Lancet Neurol. 2002, 1:232-241. 10.1016/s1474-4422(02)00102-3

15. Lauer K: Environmental risk factors in multiple sclerosis . Expert Rev Neurother. 2010, 10:421-440. 10.1586/ern.10.7

16. Sibley WA, Bamford CR, Clark K: Clinical viral infections and multiple sclerosis . Lancet. 1985, 325:13131315. 10.1016/s0140-6736(85)92801-6

17. Robbins CS, Dawe DE, Goncharova SI, et al.: Cigarette smoke decreases pulmonary dendritic cells and impacts antiviral immune responsiveness. Am J Respir Cell Mol Biol. 2004, 30:202-211. 10.1165/rcmb.200302590C

18. Bijl M, Horst G, Limburg PC, Kallenberg CGM: Effects of smoking on activation markers, Fas expression, and apoptosis of peripheral blood lymphocytes. Eur J Clin Invest. 2001, 31:550-553. 10.1046/j.13652362.2001.00842.x

19. Hedström AK, Katsoulis M, Hössjer O, et al.: The interaction between smoking and HLA genes in multiple sclerosis: replication and refinement. Eur J Epidemiol. 2017, 32:909-919. 10.1007/s10654-017-0250-2

20. Ammitzbøll C, von Essen MR, Börnsen L, et al.: GPR15+ T cells are Th17 like, increased in smokers, and associated with multiple sclerosis. J Autoimmun. 2019, 97:114-121. 10.1016/j.jaut.2018.09.005

21. Küçükali Cİ, Kürtüncü M, Çoban A, Çebi M, Tüzün E: Epigenetics of multiple sclerosis: an updated review . Neuromolecular Med. 2015, 17:83-96. 10.1007/s12017-014-8298-6

22. Didonna A, Oksenberg JR: The Genetics of Multiple Sclerosis . Multiple Sclerosis: Perspectives in Treatment and Pathogenesis. Zagon IS, McLaughlin PJ (ed): Codon Publications, Brisbane, AU; 2017. 1:3-16.

23. Hedström AK, Sundqvist E, Bäärnhielm M, et al.: Smoking and two human leukocyte antigen genes interact to increase the risk for multiple sclerosis. Brain. 2011, 134:653-664. 10.1093/brain/awq371

24. Multiple sclerosis. (2020). Accessed: May 24, 2020: https://ghr.nlm.nih.gov/condition/multiple-sclerosis.

25. Patsopoulos NA: Genetics of multiple sclerosis: an overview and new directions . Cold Spring Harb Perspect Med. 2018, 8:a028951. 10.1101/cshperspect.a028951

26. Briggs FBS, Yu JC, Davis MF, et al.: Multiple sclerosis risk factors contribute to onset heterogeneity . Mult Scler Relat Disord. 2019, 28:11-16. 10.1016/j.msard.2018.12.007

27. Briggs FBS, Acuna B, Shen L, et al.: Smoking and risk of multiple sclerosis: evidence of modification by NAT1 variants. Epidemiology. 2014, 25:605-614. 10.1097/EDE.0000000000000089

28. Marabita F, Almgren M, Sjöholm LK, et al.: Smoking induces DNA methylation changes in multiple sclerosis patients with exposure-response relationship. Sci Rep. 2017, 7:14589. 10.1038/s41598-017-14788-W

29. Rao SM, Leo GJ, Bernardin L, Unverzagt F: Cognitive dysfunction in multiple sclerosis. I. Frequency, patterns, and prediction. Neurology. 1991, 41:685-691. 10.1212/wnl.41.5.685

30. Özcan ME, İnce B, Bingöl A, et al.: Association between smoking and cognitive impairment in multiple sclerosis. Neuropsychiatr Dis Treat. 2014, 10:1715-1719. 10.2147/NDT.S68389

31. Rao SM, Grafman J, DiGiulio D, et al.: Memory dysfunction in multiple sclerosis: its relation to working memory, semantic encoding, and implicit learning. Neuropsychology. 1993, 7:364-374. 10.1037/08944105.7.3.364

32. Savettieri G, Messina D, Andreoli V, et al.: Gender-related effect of clinical and genetic variables on the cognitive impairment in multiple sclerosis. J Neurol. 2004, 251:1208-1214. 10.1007/s00415-004-0508-y

33. Borghi M, Cavallo M, Carletto S, et al.: Presence and significant determinants of cognitive impairment in a large sample of patients with multiple sclerosis. PLoS One. 2013, 8:69820. 10.1371/journal.pone.0069820

34. Inglese M, Oesingmann N, Casaccia P, Fleysher L: Progressive multiple sclerosis and gray matter pathology: an MRI perspective. Mt Sinai J Med. 2011, 78:258-267. 10.1002/msj.20247

35. Zivadinov R, Weinstock-Guttman B, Hashmi K, et al.: Smoking is associated with increased lesion volumes 
and brain atrophy in multiple sclerosis. Neurology. 2009, 73:504-510. 10.1212/WNL.0b013e3181b2a706

36. Courville CB, Maschmeyer JE, Delay CP: Effects of smoking on the acute exacerbations of multiple sclerosis . Bull Los Angel Neuro Soc. 1964, 29:1-6.

37. Healy BC, Ali EN, Guttmann CRG, et al.: Smoking and disease progression in multiple sclerosis . Arch Neurol. 2009, 66:858-864. 10.1001/archneurol.2009.122

38. Handel AE, Williamson AJ, Disanto G, Dobson R, Giovannoni G, Ramagopalan SV: Smoking and multiple sclerosis: an updated meta-analysis. PLoS One. 2011, 6:16149. 10.1371/journal.pone.0016149

39. da Silva KRP, Alvarenga RMP, Fernandez Y Fernandez O, Alvarenga H, Thuler LCS: Potential risk factors for multiple sclerosis in Rio de Janeiro: a case-control study. Arq Neuropsiquiatr. 2009, 67:229-234. 10.1590/s0004-282x2009000200011

40. Hedström AK, Hillert J, Olsson T, Alfredsson L: Smoking and multiple sclerosis susceptibility . Eur J Epidemiol. 2013, 28:867-874. 10.1007/s10654-013-9853-4

41. Pekmezovic T, Drulovic J, Milenkovic M, et al.: Lifestyle factors and multiple sclerosis: a case-control study in Belgrade. Neuroepidemiology. 2006, 27:212-216. 10.1159/000096853

42. Di Pauli F, Reindl M, Ehling R, et al.: Smoking is a risk factor for early conversion to clinically definite multiple sclerosis. Mult Scler. 2008, 14:1026-1030. 10.1177/1352458508093679

43. Hernán MA, Jick SS, Logroscino G, Olek MJ, Ascherio A, Jick H: Cigarette smoking and the progression of multiple sclerosis. Brain. 2005, 128:1461-1465. 10.1093/brain/awh471

44. Pittas F, Ponsonby A-L, van der Mei IAF, et al.: Smoking is associated with progressive disease course and increased progression in clinical disability in a prospective cohort of people with multiple sclerosis. J Neurol. 2009, 256:577-585. 10.1007/s00415-009-0120-2

45. Ramanujam R, Hedström A-K, Manouchehrinia A, Alfredsson L, Olsson T, Bottai M, Hillert J: Effect of smoking cessation on multiple sclerosis prognosis. JAMA Neurol. 2015, 72:1117-1123. 10.1001/jamaneurol.2015.1788

46. Maghzi A-H, Etemadifar M, Heshmat-Ghahdarijani K, Moradi V, Nonahal S, Ghorbani A, Minagar A: Cigarette smoking and the risk of multiple sclerosis: a sibling case-control study in Isfahan, Iran . Neuroepidemiology. 2011, 37:238-242. 10.1159/000332765

47. Sundström P, Nyström L: Smoking worsens the prognosis in multiple sclerosis . Mult Scler. 2008, 14:10311035. 10.1177/1352458508093615 